\title{
Bifunctional Janus Spheres with Chemically Orthogonal Patches
}

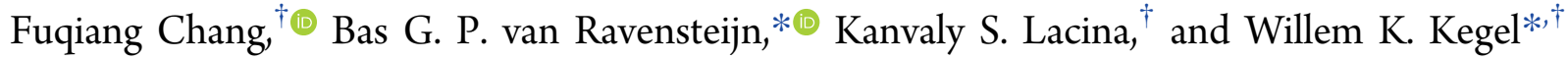 \\ ${ }^{\dagger}$ Van't Hoff Laboratory for Physical and Colloid Chemistry, Debye Institute for Nanomaterials Science, Utrecht University, 3584 \\ $\mathrm{CH}$ Utrecht, The Netherlands
}

\section{Supporting Information}

ABSTRACT: Bifunctional Janus particles with patches carrying orthogonal surface functionalities that can be independently modified are widely seen as promising building blocks for the bottom-up assembly of functional materials due to their full compositional and geometrical programmability. However, synthesis of these colloids remains an elusive task as current scalable procedures are generally limited to monofunctional particles only. Herein, a scalable bulk wet-chemical synthetic method for fabricating bifunctional Janus particles following a two-step dispersion polymerization is developed. Patch formation on these colloids is driven by the spontaneous phase separation between a brominated outer shell and poly(propargyl acrylate) ( $\mathrm{p}(\mathrm{PA})$ ), formed after the seed particles were swollen with the corresponding monomer. The size ratio between the two patches is readily tunable by controlling the volumetric ratio between the feeding monomers. The distinct patches of these Janus particles carry chemical handles facilitating independent and orthogonal surface modification using Atom Transfer Radical Polymerization (ATRP) and thiol-yne Click chemistry for the brominated and alkyne-containing patches, respectively. The presented route toward bifunctional patchy spheres provides a versatile starting point for the development of bifunctional colloidal particles with tailored directional properties.
Tan nus particles, colloidal particles consisting of two faces ("patches") of different materials that are fused together, can organize themselves into new (periodic) superstructures $^{1-3}$ with novel and potentially innovative optical, electronic, and mechanical properties. Additionally, these types of particles are the model systems of choice to study self-propulsion, ${ }^{4,5}$ can act as extremely efficient interfacial stabilizers, ${ }^{6,7}$ and are versatile immobilization platforms for (homogeneous) catalysts. ${ }^{8,9}$

Numerous methods have been developed to generate chemically anisotropic Janus particles with high uniformity, including photolithography, ${ }^{10}$ microcontact printing, ${ }^{11,12}$ microfluidic-assisted synthesis, ${ }^{13,14}$ and dip-pen nanolithography. ${ }^{15}$ By relying on emulsion or dispersion-based routes, the yields toward these colloids could be significantly improved. $^{7,16-21}$ However, these procedures typically yield particles that carry at least one patch that is chemically "inert" (monofunctional) and, hence, cannot be independently modified. ${ }^{16,20,22,23}$ This limits the achievable complexity on the single particle and superstructure level. Therefore, being able to modify both semispheres in an orthogonal fashion is expected to revolutionize the field by opening avenues to new classes of superstructures, ${ }^{24}$ new mechanisms in selfpropulsion, and to applications in, for example, "tandem" catalysis.

Inspired by the work of Zheng et al, where anisotropic colloids with chemically distinct domains were prepared, ${ }^{25}$ we present a synthetic procedure to produce uniform micrometersized Janus spheres with two chemically orthogonal modifiable

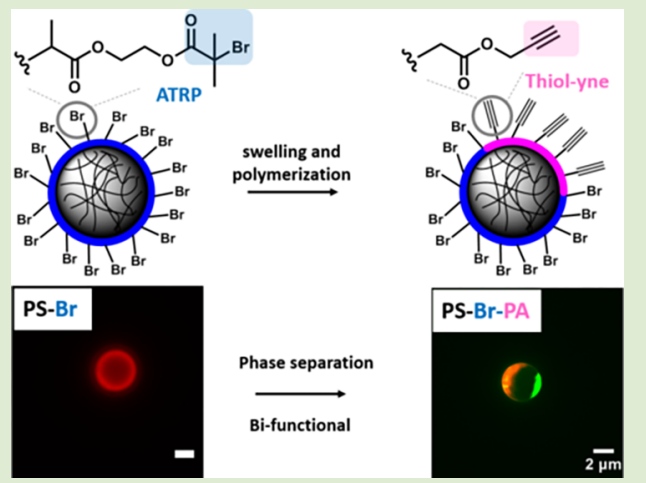

patches. Patch formation is driven by a polymerization-induced phase separation of an alkyne-containing monomer from a functional seed particle carrying ATRP initiators. After phase separation, the ATRP initiators and the propargyl acrylate (PA)-derived alkyne functionalities are confined into two welldefined domains with controllable dimensions, while the truly spherical morphology of the particles is retained. This robust synthesis of bifunctional patchy spheres provides a versatile platform for the development of new classes of colloidal particles with tailored directional properties.

As depicted in Scheme 1, the preparation of bifunctional Janus colloids was achieved via a two-step seeded dispersion polymerization. First, uniform charge-stabilized linear polystyrene (PS) microspheres $(\sim 1.7 \mu \mathrm{m}$ diameter $)$ were swollen with a monomer mixture comprising styrene $(\mathrm{St})$ and (2-(2bromoisobutyryloxy) ethyl methacrylate) (BIEM). The subsequent polymerization yields brominated core-shell particles (PS-Br), which feature a uniform spherical shape and a diameter of $1.9 \mu \mathrm{m}$ with a polydispersity of $5 \%$. Incorporation of BIEM was confirmed using infrared (IR) spectroscopy by the appearance of the characteristic $\mathrm{C}=\mathrm{O}$ signal at $1730 \mathrm{~cm}^{-1}$ (Figure S1a). Seeded polymerization as an efficient strategy to produce functional spheres with core-shell structures has been widely adapted by using (slightly) hydrophilic comonomers such as vinylbenzyl chloride, ${ }^{26} 2$ -

Received: March 15, 2019

Accepted: May 22, 2019

Published: May 28, 2019 
Scheme 1. Schematic Representation of the Synthetic Procedure toward the Janus Bifunctional Spheres ${ }^{a}$

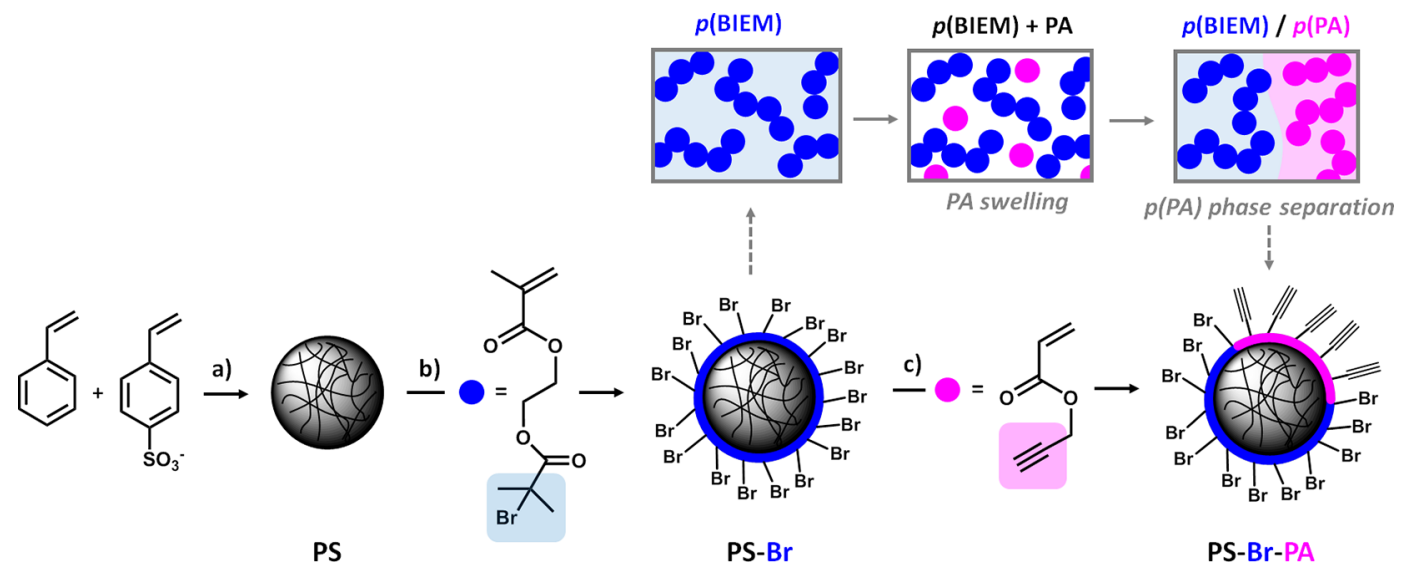

${ }^{a}$ Step (a): preparation of polystyrene seeds (PS) by stabilizer-free dispersion polymerization of styrene (St) and sodium styrenesulfonate (NaSS). Step (b): synthesis of brominated core-shell particles (PS-Br) via a seeded dispersion polymerization using a 2-(2-bromoisobutyryloxy) ethyl methacrylate (BIEM, blue circle) containing a monomer mixture. Step (c): synthesis of bifunctional Janus spheres (PS-Br-PA) by swelling of PS-Br with a propargyl acrylate (PA, magenta circle) containing monomer mixture and subsequent polymerization of the swelling monomers. Patch formation is driven by a polymerization-induced phase separation between the $\mathrm{p}$ (BIEM)-rich polymers (connected blue circles) and the newly formed $\mathrm{p}(\mathrm{PA})$ (connected magenta circles), as schematically depicted on the top of the figure.

(2-bromoisobutyryloxy) ethyl acrylate, ${ }^{27}$ or trimethoxysilylpropyl acrylate ${ }^{20}$ that prefer to partition on the periphery of the colloids. 4-Vinylbenzenesulfonate (NaSS) was employed as a charged comonomer. The permanently charged sulfonate group will mainly be exposed to the aqueous dispersing medium, providing electrostatic repulsion driven colloidal stability. In contrast to conventional dispersion polymerizations, which rely on steric stabilization via surfaceadsorption of polymers, the use of NaSS safeguards easy accessibility of the surface-immobilized functional handles facilitating further modification procedures as will be discussed later.

To probe the distribution of functional groups over the particle surface, the PS-Br core-shell particles were grafted with poly(3-(trimethoxysilyl) propyl methacrylate) (p(TPM)) brushes via ATRP and subsequent fluorescent labeling of the immobilized polymers (Figure 1a). Fluorescence microscopy images confirmed a homogeneous distribution of the dyes, and therefore BIEM, over the surface of the core-shell spheres (Figure 1b).

Next, the brominated seed particles were swollen with a mixture of St, propargyl acrylate (PA), NaSS, and nonpolymerizable toluene. PA was selected as comonomer for the second seeded dispersion polymerization, since this monomer has an appreciable miscibility with PS, while the corresponding polymer poly(propargyl acrylate) does not. ${ }^{16}$ Therefore, we hypothesize that a phase separation is induced during the PA polymerization, leading to the desired bifunctional Janus spheres. Additionally, the alkyne groups of $\mathrm{p}(\mathrm{PA})$ are an attractive functional handle for postcolloidal synthetic modifications, as will be shown later. To facilitate phase separation of the newly formed $\mathrm{p}(\mathrm{PA})$ from the seed particles, toluene was added during the second swelling step. As reported before for similar systems, toluene primarily acts as a plasticizer, increasing polymer flexibility by lowering the viscosity of the colloidal interior. ${ }^{28,29}$ In the absence of toluene, phase separation of $\mathrm{p}(\mathrm{PA})$ proved to be incomplete, resulting in the formation of multiple $\mathrm{p}(\mathrm{PA})$ domains instead of one well-defined patch (Figure S5b).
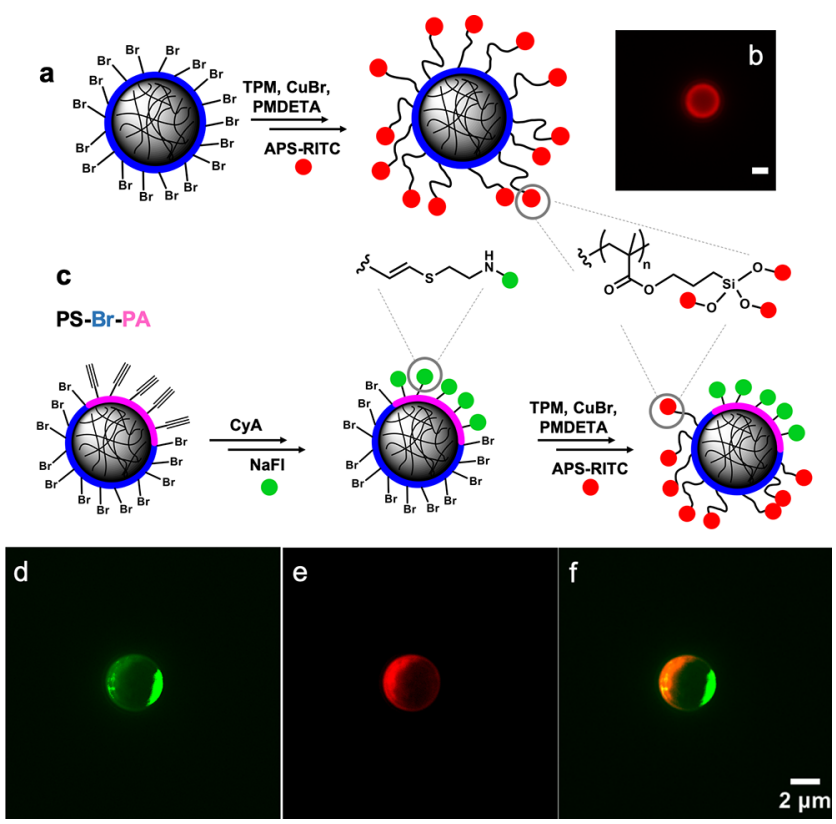

Figure 1. (a) Surface modification of PS-Br and (b) the fluorescence image of APS-RITC labeled PS-Br (scale bar $=1 \mu \mathrm{m})$. (c) The surface of PS-Br-PA was sequentially modified using ATRP and thiol-yne Click chemistry as orthogonal functionalization procedures and labeled with corresponding dyes, respectively. Fluorescence microscopy images of (d) Janus spheres solely functionalized through a thiol-yne reaction with CyA and subsequent labeling with NaFl. (e) Janus spheres solely functionalized via ATRP and subsequent labeling with APS-RITC and (f) PS-Br-PA after sequential labeling.

Since patch formation is strictly driven by polymerizationinduced phase separation from non-cross-linked PS-Br colloids, the resulting bifunctional colloids were anticipated to be spherical. In contrast, swelling and subsequent heatinduced polymerization from cross-linked spherical seeds typically results in frequently reported anisotropic particles. $^{30-32}$ As concluded from SEM analysis (Figure 2a), the resulting particles indeed feature a uniform spherical shape 


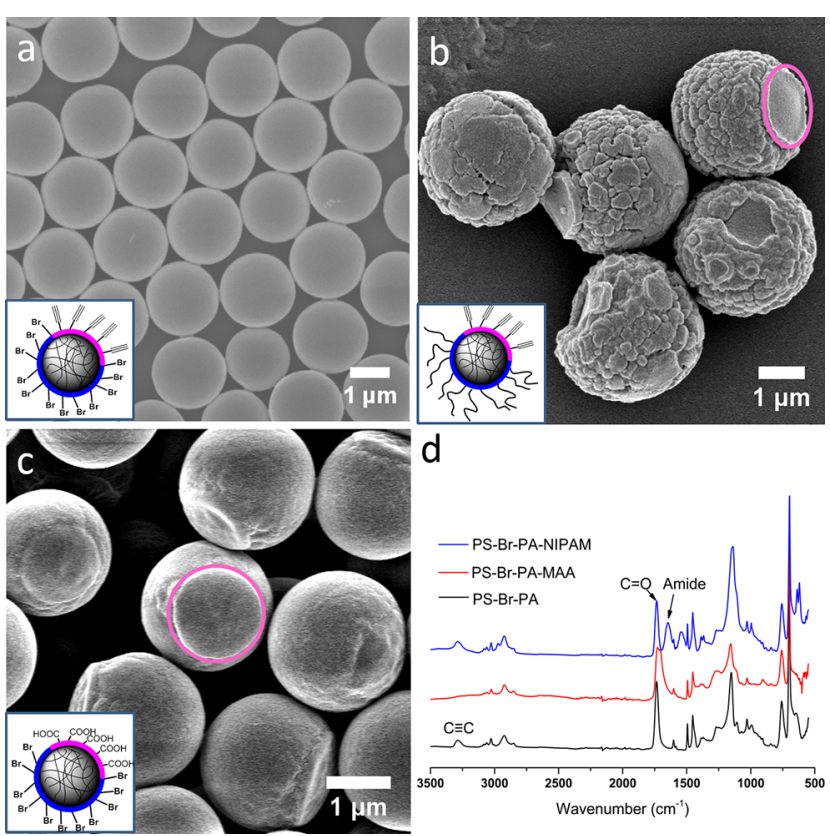

Figure 2. SEM images of PS-Br-PA (a) before modification, (b) after ATRP of NIPAM (the unmodified $\mathrm{p}$ (PA) patch is highlighted by the magenta circle), (c) after thiol-yne Click reaction with MAA (the modified $\mathrm{p}(\mathrm{PA})$ patch is highlighted by the magenta circle). (d) IR spectra of PS-Br-PA colloids shown in panels a-c.

with a smooth surface. Successful incorporation of the PA was confirmed by IR spectroscopy (Figure 2d) from the appearance of the diagnostic acetylene vibrations located at $3270 \mathrm{~cm}^{-1}$.

To map the surface distribution of the chemically functional handles on PS-Br-PA, the particles were labeled via two orthogonal approaches. The $\mathrm{p}(\mathrm{PA})$ patches were first modified via a thiol-yne Click reaction with cysteamine (CyA) to install a surface-exposed primary amine (section S1.5). Subsequently, fluorescein sodium salt $(\mathrm{NaFl})$ was coupled to the PA-derived patch relying on a 1-ethyl-3-(3-(dimethylamino)propyl)carbodiimide hydrochloride (EDC) coupling between the NaFl's carboxylate functionalities and the surface immobilized amines. Fluorescence microscopy revealed that one small region of the particles was labeled (Figure 1d), confirming the localization of the amine and, therefore, alkyne functionalities into a well-defined patch. Employing PS-Br-PA without pretreatment with CyA yielded no fluorescence signal, strengthening our hypothesis that the labeled domain corresponds to the amine-modified PA patch (Figure S4e).

Leveraging the fact that the two chemistries selected to label the brominated and alkyne-containing patch are orthogonal, the PS-Br-PA particles with a NaFl-labeled alkyne-patch were exposed to the labeling procedure previously employed for PS$\mathrm{Br}$ (Figure 1a). The results of this sequential labeling are depicted in Figure 1. Overlaying the two imaging channels clearly reveals that the fluorescence intensity is distributed over two distinct patches that are located at opposite sides of the colloids (Figure 1f). Therefore, these labeling experiments highlight two key features of the presented Janus colloids: (1) the particles carry two well-defined patches, in agreement with our hypothesis that PA is able to undergo polymerizationinduced phase separation from the brominated core-shell particles to drive patch formation; (2) The orthogonal surface chemistries, that is, alkynes and ATRP initiators, of these patches allow for independent, site-specific surface modifications. Relying on wet-chemical procedures only, these complex particles are accessible in large quantities. Approximately $10^{10}$ particles per $\mathrm{mL}$ of dispersion are formed and the procedure is scalable up to a total volume of $50 \mathrm{~mL}$.

With the orthogonal, bifunctional Janus particles in hand, we set out to demonstrate the versatility of the presented functionalization strategies. To this end, the brominated patches were grafted with two other polymers in addition to the previously discussed $\mathrm{p}$ (TPM), namely, poly(2-hydroxyethyl acrylate) ( $\mathrm{p}(\mathrm{HEA}))$ and poly $(\mathrm{N}$-isopropylacrylamide) $(\mathrm{p}(\mathrm{NIPAM}))$ to yield hydrophilic and thermosensitive polymer brushes, respectively. Successful grafting of $p($ HEA) was confirmed from the increase in the signal intensity of the $\mathrm{C}=\mathrm{O}$ vibration (red spectrum in Figure $\mathrm{S} 1 \mathrm{~b}$ ) caused by the pendant ester moieties in the repeating units of the immobilized $\mathrm{p}$ (HEA) polymers. While successful grafting of $\mathrm{p}$ (NIPAM) was confirmed by the presence of amide vibrational signals attributed to the repeating units of $\mathrm{p}$ (NIPAM) (blue spectrum in Figure 2d). In addition to this spectroscopic evidence, SEM images revealed enhanced surface roughness on the reactive brominated lobes of the Janus spheres after polymer grafting (Figure $2 \mathrm{~b}$ ). This roughness is caused by a collapse of the polymer brushes during sample preparation for electron microscopy. The unmodified PA patch remained smooth and intact (Figure 2b, pink circles), highlighting the site-specificity of the polymer grafting.

Modification of the $\mathrm{p}(\mathrm{PA})$ patch was shown through thiolyne Click chemistry with mercaptoacetic acid (MAA). Functionalization with MAA was confirmed from the disappearance of the alkyne vibration at $3270 \mathrm{~cm}^{-1}$ (red spectrum, Figure 2d). Moreover, SEM analysis of the colloids after site-selective modification with MAA revealed a transition of a perfectly smooth spherical colloid to hazelnut-shaped particles (Figure 2c). This morphological transition can be rationalized by considering that coupling of MAA leads to a significant enhancement in the hydrophilicity of the $\mathrm{p}(\mathrm{PA})$ patch. As a result, the MAA-functionalized patch swells, making it appear as a protruding cap on the particle surface.

Furthermore, we demonstrated that the ATRP and Click modifications could be performed in a sequential fashion on the same batch of colloids (Figure 3a). The order of sequential modification, which enables tunability of physical and chemical properties of both patches simultaneously, proved to be irrelevant. For example, the $\mathrm{p}(\mathrm{PA})$ patches of PS-Br-PA could be modified via a thiol-yne Click reaction with MAA (the disappearance of the alkyne vibration at $3270 \mathrm{~cm}^{-1}$ in the red spectrum of Figure $3 \mathrm{~b}$ ) and subsequently grafted with HEA $\left(\mathrm{C}=\mathrm{O}\right.$ vibration at $1732 \mathrm{~cm}^{-1}$ in the blue spectrum of Figure 3b). Alternatively, grafting of NIPAM (red spectrum of Figure 3c), followed by a thiol-yne coupling of MAA, was successful as well as concluded from the presence of $\mathrm{O}-\mathrm{H}$ stretching vibrations at $3300-2600 \mathrm{~cm}^{-1}$ (Figure $3 \mathrm{c}$, blue spectrum). Prerequisite for this last route is that the grafted polymers do not sterically hinder the accessibility of the $\mathrm{p}(\mathrm{PA})$ patch for further modification, as would be the case for long immobilized polymer chains. Additional examples to claim orthogonality and versatility of the surface modifications can be found in Supporting Information S7 and Figure S6. After the surface modifications, the Janus spheres remained readily dispersible in water, methanol, and ethanol (Figure S7), promoting their use colloidal building blocks in future applications. 

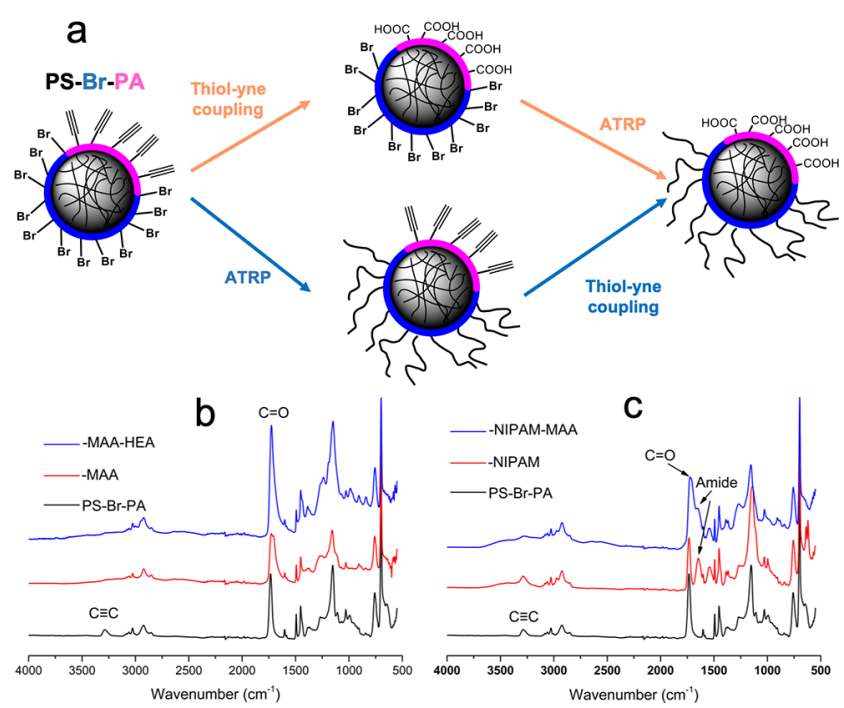

Figure 3. (a) Schematic representation of the sequential modification of PS-Br-PA via two different routes. IR spectra of PS-Br-PA modified via (b) thiol-yne Click reaction with MAA followed by grafting of a $\mathrm{p}$ (HEA) polymer brush, and (c) grafting of $\mathrm{p}$ (NIPAM) followed by a thiol-yne Click reaction with MAA (see S1.5 and S1.6 for more details).

Leveraging the fact that surface modifications with MAA leads to morphological alternations of the $\mathrm{p}(\mathrm{PA})$ patch, we investigated the scope of our colloidal synthesis in terms of patch size. Theoretically, the size of the $\mathrm{p}(\mathrm{PA})$ domain should scale with the volumetric ratio of the swelling monomer solution (St and PA) to the brominated core-shell seed particles $\left(V_{\mathrm{S}}=V_{\text {monomers }} / V_{\text {core-shell }}\right)$ used in the second seeded dispersion polymerization (Figure 1 , step $b$ ). ${ }^{21}$ This geometric control over the patch dimensions was indeed confirmed by employing a series of swelling ratios ranging from 0.8 to 3 . Within this window, PS-Br-PA colloids with a patch size ratio, here defined as the surface of the PA patch divided by the surface of the brominated patch $\left(S_{\text {PA-patch }} / S_{\text {PS-Br-PA }}\right)$, of 0.09 to 0.74 were achievable (Figure $4 \mathrm{a}-\mathrm{c}$ ). This tunability of the patch size, as probed with SEM, was corroborated by fluorescent microscopy, as the sizes of the fluorescently labeled $\mathrm{p}(\mathrm{PA})$ patches are directly proportional with the employed swelling ratio (Figure $4 a-c$, inset). Synthesis of PS-Br-PA colloids carrying even larger $\mathrm{p}(\mathrm{PA})$ patches proved to be unfeasible. At swelling ratios $>2$, the added monomer (partially) dissolves the polystyrene core during the swelling procedure, leading to irregularly shaped and collapsed particles after polymerization (Figure $4 \mathrm{~d}$ ).

In conclusion, this study has introduced an efficient and scalable approach for the synthesis of uniform Janus particles with two chemically orthogonal patches carrying ATRP initiators and alkyne functionalities. The Janus particles with tunable patch ratio were realized via a two-step seeded dispersion polymerization. PS template spheres were uniformly coated with a functional brominated shell containing ATRP initiators. Swelling these particles with alkyne-functionalized monomer (PA), followed by polymerization, triggers phase separation between the newly formed $\mathrm{p}(\mathrm{PA})$ and the brominated shell, yielding the bifunctional Janus colloids. The biphasic functionality of the Janus spheres was demonstrated by (sequential) patch modification using Click chemistry and ATRP. Ultimately, we expect that these particles will serve as a versatile starting point for the synthesis of
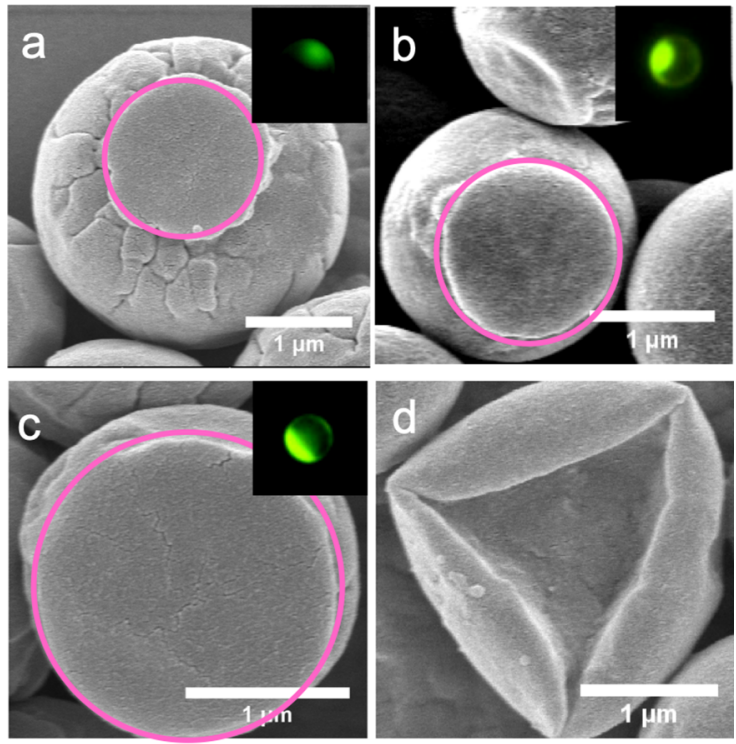

e

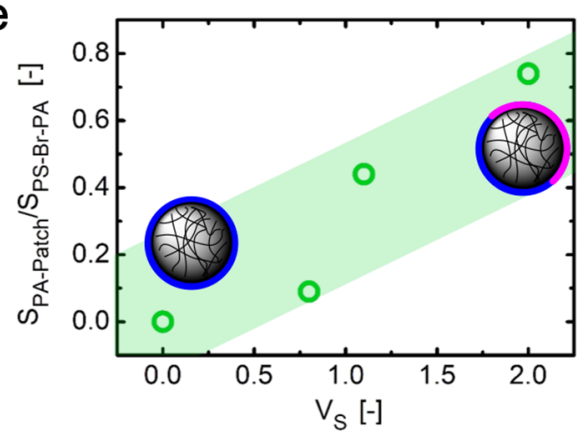

Figure 4. SEM images of MAA-modified Janus particles with different patch ratios $\left(S_{\text {PA-patch }} / S_{\text {PS-Br-PA }}\right),($ a $) 0.09\left(V_{\mathrm{S}}=0.8\right)$, (b) $0.44\left(V_{\mathrm{S}}=\right.$ $1.1)$, and $(c) 0.74\left(V_{S}=2\right)$. The modified PA region are highlighted with magenta circles. Insets: corresponding fluorescence microscopy images of Janus spheres functionalized using with CyA and subsequent labeling with $\mathrm{NaFl}$. (d) Collapsed PS-Br-PA at $V_{\mathrm{S}}=3$ (e). Dependence of $S_{\text {PA-patch }} / S_{\text {PS-Br-PA }}$ on the swelling ratio employed in the second seeded dispersion polymerization (Scheme 1, step b), as determined by SEM.

tailored multifunctional building blocks. The unique capability to orthogonally tune the properties of well-defined regions on a single colloidal particle will provide new opportunities in the fields of directed colloidal assembly, multiresponsive selfpropelling particles, and (tandem) catalysis.

\section{ASSOCIATED CONTENT}

\section{S Supporting Information}

The Supporting Information is available free of charge on the ACS Publications website at DOI: 10.1021/acsmacrolett.9b00193.

Synthetic and characterization details, including Figures S1-S7 (PDF)

\section{AUTHOR INFORMATION}

\section{Corresponding Authors}

*E-mail: w.k.kegel@uu.nl.

*E-mail: bas.van.ravensteijn@gmail.com.

ORCID

Fuqiang Chang: 0000-0001-8811-8393 
Bas G. P. van Ravensteijn: 0000-0001-9024-3927

Notes

The authors declare no competing financial interest.

\section{ACKNOWLEDGMENTS}

We acknowledge funding from the CSC No. 201406230043. Michele Zanini is thanked for comments on the manuscript.

\section{REFERENCES}

(1) Chen, Q.; Bae, S. C.; Granick, S. Directed Self-Assembly of a Colloidal Kagome Lattice. Nature 2011, 469 (7330), 381-384.

(2) Chen, Q.; Whitmer, J. K.; Jiang, S.; Bae, S. C.; Luijten, E.; Granick, S. Supracolloidal Reaction Kinetics of Janus Spheres. Science (Washington, DC, U. S.) 2011, 331 (6014), 199-202.

(3) Evers, C. H. J.; Luiken, J. A.; Bolhuis, P. G.; Kegel, W. K. SelfAssembly of Microcapsules via Colloidal Bond Hybridization and Anisotropy. Nature 2016, 534 (7607), 364-368.

(4) Wang, H.; Pumera, M. Fabrication of Micro/Nanoscale Motors. Chem. Rev. 2015, 115 (16), 8704-8735.

(5) Singh, D. P.; Choudhury, U.; Fischer, P.; Mark, A. G. NonEquilibrium Assembly of Light-Activated Colloidal Mixtures. Adv. Mater. 2017, 29 (32), 1701328.

(6) Kim, J.-W.; Lee, D.; Shum, H. C.; Weitz, D. a. Colloid Surfactants for Emulsion Stabilization. Adv. Mater. 2008, 20 (17), 3239-3243.

(7) Tu, F.; Lee, D. Shape-Changing and Amphiphilicity-Reversing Janus Particles with PH-Responsive Surfactant Properties. J. Am. Chem. Soc. 2014, 136 (28), 9999-10006.

(8) Tan, H.; Guo, S.; Dinh, N.-D.; Luo, R.; Jin, L.; Chen, C.-H. Heterogeneous Multi-Compartmental Hydrogel Particles as Synthetic Cells for Incompatible Tandem Reactions. Nat. Commun. 2017, 8 (1), 663.

(9) Yang, T.; Wei, L.; Jing, L.; Liang, J.; Zhang, X.; Tang, M.; Monteiro, M. J.; Chen, Y. I.; Wang, Y.; Gu, S.; Zhao, D.; Yang, H.; Liu, J.; Lu, G. Dumbbell-Shaped Bi-Component Mesoporous Janus Solid Nanoparticles for Biphasic Interface Catalysis. Angew. Chem., Int. Ed. 2017, 56 (29), 8459-8463.

(10) Vogel, N.; Retsch, M.; Fustin, C.-A.; del Campo, A.; Jonas, U. Advances in Colloidal Assembly: The Design of Structure and Hierarchy in Two and Three Dimensions. Chem. Rev. 2015, 115 (13), 6265-6311.

(11) Li, M.; Li, W. H.; Zhang, J.; Alici, G.; Wen, W. A Review of Microfabrication Techniques and Dielectrophoretic Microdevices for Particle Manipulation and Separation. J. Phys. D: Appl. Phys. 2014, 47 (6), 063001.

(12) Kaufmann, T.; Gokmen, M. T.; Rinnen, S.; Arlinghaus, H. F.; Du Prez, F.; Ravoo, B. J. Bifunctional Janus Beads Made by "Sandwich" Microcontact Printing Using Click Chemistry. J. Mater. Chem. 2012, 22 (13), 6190-6199.

(13) Park, J. Il; Saffari, A.; Kumar, S.; Günther, A.; Kumacheva, E. Microfluidic Synthesis of Polymer and Inorganic Particulate Materials. Annu. Rev. Mater. Res. 2010, 40 (1), 415-443.

(14) Rahmani, S.; Saha, S.; Durmaz, H.; Donini, A.; Misra, A. C.; Yoon, J.; Lahann, J. Chemically Orthogonal Three-Patch Microparticles. Angew. Chem., Int. Ed. 2014, 53 (9), 2332-2338.

(15) Gentili, D.; Cavallini, M. Wet-Lithographic Processing of Coordination Compounds. Coord. Chem. Rev. 2013, 257 (17-18), 2456-2467.

(16) Bradley, L. C.; Stebe, K. J.; Lee, D. Clickable Janus Particles. J. Am. Chem. Soc. 2016, 138 (36), 11437-11440.

(17) Chen, W.-H.; Tu, F.; Bradley, L. C.; Lee, D. Shape-Tunable Synthesis of Sub-Micrometer Lens-Shaped Particles via Seeded Emulsion Polymerization. Chem. Mater. 2017, 29 (7), 2685-2688.

(18) Kim, J. W.; Cho, J.; Cho, J.; Park, B. J.; Kim, Y.-J.; Choi, K.-H.; Kim, J. W. Synthesis of Monodisperse Bi-Compartmentalized Amphiphilic Janus Microparticles for Tailored Assembly at the OilWater Interface. Angew. Chem. 2016, 128 (14), 4585-4589.
(19) Li, B.; Wang, M.; Chen, K.; Cheng, Z.; Chen, G.; Zhang, Z. Synthesis of Biofunctional Janus Particles. Macromol. Rapid Commun. 2015, 36 (12), 1200-1204.

(20) Park, J.-G.; Forster, J. D.; Dufresne, E. R. High-Yield Synthesis of Monodisperse Dumbbell-Shaped Polymer Nanoparticles. J. Am. Chem. Soc. 2010, 132 (17), 5960-5961.

(21) Mock, E. B.; De Bruyn, H.; Hawkett, B. S.; Gilbert, R. G.; Zukoski, C. F. Synthesis of Anisotropic Nanoparticles by Seeded Emulsion Polymerization. Langmuir 2006, 22 (9), 4037-4043.

(22) Zhu, H.; Nagaoka, Y.; Hills-Kimball, K.; Tan, R.; Yu, L.; Fang, Y.; Wang, K.; Li, R.; Wang, Z.; Chen, O. Pressure-Enabled Synthesis of Hetero-Dimers and Hetero-Rods through Intraparticle Coalescence and Interparticle Fusion of Quantum-Dot-Au Satellite Nanocrystals. J. Am. Chem. Soc. 2017, 139 (25), 8408-8411.

(23) Van Ravensteijn, B. G. P.; Kamp, M.; Van Blaaderen, A.; Kegel, W. K. General Route toward Chemically Anisotropic Colloids. Chem. Mater. 2013, 25 (21), 4348-4353.

(24) Zhang, S.; Li, Z.; Samarajeewa, S.; Sun, G.; Yang, C.; Wooley, K. L. Orthogonally Dual-Clickable Janus Nanoparticles via a Cyclic Templating Strategy. J. Am. Chem. Soc. 2011, 133 (29), 1104611049.

(25) Zheng, X.; Wang, Y.; Wang, Y.; Pine, D. J.; Weck, M. Thermal Regulation of Colloidal Materials Architecture through Orthogonal Functionalizable Patchy Particles. Chem. Mater. 2016, 28 (11), 39843989.

(26) van Ravensteijn, B. G. P.; Kegel, W. K. Tuning Particle Geometry of Chemically Anisotropic Dumbbell-Shaped Colloids. J. Colloid Interface Sci. 2017, 490, 462-477.

(27) Van Ravensteijn, B. G. P.; Hendriksen, W. E.; Eelkema, R.; Van Esch, J. H.; Kegel, W. K. Fuel-Mediated Transient Clustering of Colloidal Building Blocks. J. Am. Chem. Soc. 2017, 139 (29), 97639766.

(28) Wang, S.; Wu, N. The Impact of Nonpolymerizable Swelling Agents On The Synthesis of Particles With Combined Geometric, Interfacial, and Compositional Anisotropy. Langmuir 2015, 31 (29), $7962-7969$.

(29) Yang, M.; Wang, G.; Ma, H. An Efficient Approach for Production of Polystyrene/Poly(4-Vinylpridine) Particles with Various Morphologies Based on Dynamic Control. Chem. Commun. 2011, 47 (3), 911-913.

(30) Kim, J.; Larsen, R. J.; Weitz, D. a. Synthesis of Nonspherical Colloidal Particles with Anisotropic Properties. J. Am. Chem. Soc. 2006, 128 (44), 14374-14377.

(31) Kim, J.-W.; Larsen, R. J.; Weitz, D. A. Uniform Nonspherical Colloidal Particles with Tunable Shapes. Adv. Mater. 2007, 19 (15), 2005-2009.

(32) Kraft, D. J.; Hilhorst, J.; Heinen, M. a P.; Hoogenraad, M. J.; Luigjes, B.; Kegel, W. K. Patchy Polymer Colloids with Tunable Anisotropy Dimensions. J. Phys. Chem. B 2011, 115 (22), 7175-7181. 